\title{
Procedure to Solve Network DEA Based on a Virtual Gap Measurement Model
}

\author{
Fuh-hwa Franklin Liu and Yu-cheng Liu \\ Department of Industrial Engineering \& Management, National Chiao Tung University, Hsinchu, Taiwan \\ Correspondence should be addressed to Yu-cheng Liu; brady.liu@mtigroup.com
}

Received 15 October 2016; Accepted 10 January 2017; Published 15 February 2017

Academic Editor: Hiroyuki Mino

Copyright (C) 2017 Fuh-hwa Franklin Liu and Yu-cheng Liu. This is an open access article distributed under the Creative Commons Attribution License, which permits unrestricted use, distribution, and reproduction in any medium, provided the original work is properly cited.

\begin{abstract}
Network DEA models assess production systems that contain a set of network-structured subsystems. Each subsystem has input and output measures from and to the external network and has intermediate measures that link to other subsystems. Most published studies demonstrate how to employ DEA models to establish network DEA models. Neither static nor dynamic network DEA models adjust the links. This paper applies the virtual gap measurement (VGM) model to construct a mixed integer program to solve dynamic network DEA problems. The mixed integer program sets the total numbers of "as-input" and "as-output" equal to the total number of links in the objective function. To obtain the best-practice efficiency, each DMU determines a set of weights for inputs, outputs, and links. The links are played either "as-input" or "as-output." Input and as-input measures reduce slack, whereas output and as-output measures increase slacks to attain their target on the production frontier.
\end{abstract}

\section{Introduction}

Data envelopment analysis (DEA) models are used to measure the relative efficiency of each decision-making unit $(\mathrm{DMU})$ relative to its peers regarding multiple input indices versus multiple output indices. Fewer input values and more output values are desired to improve the model's aggregated performance score. The DMU assigns a set of weights to the indices to obtain the best-practice performance score. The drawback of these models is the omission of the internal processes between inputs and outputs.

Seiford and Zhu [1] developed a DEA approach for evaluating US commercial banks in a two-stage process characterized by profitability and marketability. Zhu [2] applied the same two-stage process to the Fortune Global 500 companies. Sexton and Lewis [3] studied the performance of Major League Baseball in a two-stage process. The above twostage DEA papers are among the first to address formally the links between the two internal stages and the link categories being distinguished and discussed; these are the origin of network DEA. For a two-stage model, the links' role implies a reduction in the first-stage outputs, thereby reducing the efficiency of that stage but increasing the efficiency of the second stage. A number of DEA studies have been developed in an attempt to address this type of conflict. For instance, Lewis and Sexton [4] used the network DEA approaches of Färe and Whittaker [5] and Färe and Grosskopf [6, 7] to compute the efficiency scores of subprocesses. "Link" cannot be adjusted freely in radial models, which adjust the inputs and outputs by the efficiency scores in a twostage process. For these models, the entire system efficiency cannot be improved by adjusting links; see Kao and Hwang [8] and Lewis and Sexton [4]. "Link" applied in nonradial models (SBM), as has been discussed in recent years. Tone and Tsutsui [9] introduced a network DEA and categorized links into two types of "fixed links" and "free links." "Free links" means that the intermediate items are adjustable or discretionary, not to change direction, and each DMU can be increased or decreased from the observed one and is free to assign each individual link to one of the three characteristics: as-input, as-output, or nondiscretionary so that entire system efficiency could be maximized. "Fixed links" means that the intermediate items are nonadjustable or nondiscretionary. The linking activities are kept unchanged that cannot improve the efficiency of the entire system. In other words, the intermediate products are beyond the control of DMUs. 
The recent extension of network DEA development to dynamic DEA does not represent the effect of carry-over activities (links) between two consecutive terms (nodes). Tone and Tsutsui [10] and Kao [11] introduced the dynamic DEA model using the nonradial and radial DEA models, respectively. These researchers express the outputs from term " $t$ " as being carried over to term " $t+1$," which means that the carry-over can be regarded as one type of link. Tone and Tsutsui [12] categorized carry-over into four types: desirable (good), undesirable (bad), discretionary (free), and nondiscretionary (fixed). The discretionary link is the same as the free link in network DEA. Kao [13] considered general multistage systems in which exogenous inputs are consumed in addition to intermediate products. Cheng and Gao [14] proposed a matrix-type network model which uses data in input-output tables; it is tested and can be feasible in evaluating the relative performance. There are many articles illustrating the application of free links and fixed links. Lozano [15] relaxed the constraints for both the fixed-link and the freelink cases, thus enhancing the discriminating power of the model. This study is to present the performance assessment of the individual processes of an external efficiency model. Avkiran [16] illustrated dynamic network data envelopment analysis (DN-DEA) in commercial banking with emphasis on testing robustness. Huang et al. [17] proposed a two-stage network model with bad outputs and supper efficiency (USNSBM). Empirical comparisons show that the US-NSBM may be promising and practical for taking the nonperforming loans into account and being able to rank all samples.

Decreasing the volumes of inputs and "as-input" links and increasing the volumes of outputs and "as-output" links would improve the entire system efficiency of $\mathrm{DMU}_{o}$. Tone and Tsutsui [10] introduced a slack-based measurement (SBM) ex-post approach (adjusted score) and a 0-1 mixed integer fractional program (MIP) to address the discretionary slacks. The MIP model is, in fact, a nonlinear program. Therefore, a $0-1$ binary decision variable $\delta_{i t}$ is assigned to free link $i$ at term $t$, transforming the process into a SBM model. These links introduced objective function in the MIP model introduced by Tone and Tsutsui [10], which is the ratio of inputs and as-input links to output and as-output links. The symbol $n$ free is the total number of free links. We suggest the upper bounds of the furthest right summations in the numeration and denomination should be replaced by $n$ free ${ }^{-}$ and $n$ free $^{+}$, respectively. The nonradial model SBM has the advantage of determining the slack on each input, output, and free link. However, this model requires the total number of as-input and as-output free links to generate the aggregate efficiency score.

The current paper adopts the virtual gap measurement (VGM) model introduced by Liu and Huang [18]. The prime form of VGM is to seek the minimum virtual gap instead of the maximal efficiency score. The obtained optimal values of dual variables are used to compute the final efficiency score. The prime and dual models are well defined and explainable and the analysis is reliable. The contribution of this paper is to solve the network DEA problem raised in Tone and Tsutsui [10]. We employ a VGM two-phase procedure and linear integer restrictions, which were developed by Cook and
Zhu [19]. The researchers adopted linear integer restrictions to capture the nonlinear expression, without actually having to specify it directly in the optimization model. This paper introduces a two-phase approach to solve the problem. PhaseI is a mixed integer program model to partition the links into two sets: as-input and as-output. Phase-II is a linear program model to determine the slack of each input, as-input link, output, and as-output link. The best-practice aggregated efficiency of $\mathrm{DMU}_{o}$ in the entire system was obtained. The slacks of inputs, outputs, and free links are obtainable. The VGM model is presented in Section 2. The proposed two-phase performance evaluation model is presented in Section 3. Because the uniqueness of the optimal solution is important, we present an experiment on this subject in Section 4. Managerial insights are introduced in Section 5. We conclude this paper in the last section.

\section{Virtual Gap Measurement Model}

A set of DMUs $J=\{1,2, \ldots, n\}$ uses a production technology that transforms a set of inputs $I=\{1,2, \ldots, m\}$ into a set of outputs $R=\{1,2, \ldots, s\}$. Let the notions $x_{i j}$ and $y_{r j}$ be the nonnegative volumes of inputs and outputs of $\mathrm{DMU}_{j}$. The VGM DEA model is to measure the maximum efficiency score of $\mathrm{DMU}_{o}$. The multiplier (dual) form of the model depicts the objective function as minimizing the virtual gap $\left(\Delta_{o}^{*}\right)$ between virtual-input $\left(\sum_{i \in I} x_{i o} v_{i}\right)$ and virtual-output $\left(\sum_{r \in R} y_{r o} u_{r}\right)$. Each DMU in set $J$ alternatively acts as $\mathrm{DMU}_{o}$. Let the symbol " $\theta$ " denote the commensurate virtual unit that is used for virtual gap, virtual-input, and virtual-output. The first set of constraints ensures all DMUs have a nonnegative virtual gap. The vectors $v=\left(v_{1}, v_{2}, \ldots, v_{m}\right)$ and $u=\left(u_{1}, u_{2}, \ldots, u_{s}\right)$ are the decision variables of the weights to be assigned to the inputs and outputs. The second and third constraints define that each weighted input and output have a lower bound $\tau^{\prime}$, a constant value with unit of $\emptyset$. These assign dual variables $\pi_{i}, q_{i}$, and $p_{r}$ to the three constraints. The envelopment (prime) model of VGM is [M1]:

$$
\begin{aligned}
\Delta_{o}^{*}=\min & \left(\sum_{i \in I} x_{i o} v_{i}^{\prime}-\sum_{r \in R} y_{r o} u_{r}^{\prime}\right), \quad o=1,2, \ldots, n ; \\
\text { s.t. } \quad & \sum_{i \in I} x_{i j} v_{i}^{\prime}-\sum_{r \in R} y_{r j} u_{r}^{\prime} \geq 0, \quad j \in J \\
& x_{i o} v_{i}^{\prime} \geq \tau^{\prime}, \quad i \in I \\
& y_{r o} u_{r}^{\prime} \geq \tau^{\prime}, \quad r \in R \\
& v_{i}^{\prime} \geq 0, \quad i \in I \\
& u_{r}^{\prime} \geq 0, \quad r \in R .
\end{aligned}
$$

The dual to model [M1] can be expressed as [M2]:

$$
\begin{aligned}
\delta_{o}^{*}=\max & \tau^{\prime}\left(\sum_{i \in I} \frac{q_{i}}{x_{i o}}+\sum_{r \in R} \frac{p_{r}}{y_{r o}}\right) \\
\text { s.t. } & \sum_{j \in J} \pi_{j} x_{i j}=x_{i o}-q_{i}, \quad i \in I ;
\end{aligned}
$$




$$
\begin{aligned}
& \sum_{j \in J} \pi_{j} y_{r j}=y_{r o}-p_{r}, \quad r \in R ; \\
& \pi_{j} \geq 0, \quad j \in J ; \\
& q_{i} \geq 0, \quad i \in I ; \\
& p_{r} \geq 0, \quad r \in R .
\end{aligned}
$$

The decision variable $\pi_{j}$ denotes the weight of $\mathrm{DMU}_{j}$. $q_{i}$ and $p_{r}$ denote the slacks of $i$ th input and $r$ th output, respectively. The objective function (7) expresses the maximum summation of improvement ratios of inputs and outputs. Set $\tau^{\prime}=1$ temporarily for computation convenient, and it will not affect the optimal solutions. The decision variable with a superscript “*” denotes its optimal value. The following equation defines the constant value of $\tau$; it is equal to the reciprocal of the maximum virtual-inputs of DMUs:

$$
\tau=\frac{1}{\max _{j \in J} \sum_{i \in I} x_{i j} v_{i}^{\prime *}} .
$$

Thus, the optimal solutions are normalized as

$$
\begin{aligned}
& v_{i}^{*}=v_{i}^{\prime *} \times \tau, \quad i \in I ; \\
& u_{r}^{*}=u_{r}^{\prime *} \times \tau, \quad r \in R .
\end{aligned}
$$

Evaluating different $\mathrm{DMU}_{o}$ 's, one may directly compare their weights, virtual gap, virtual-input, and virtual-output vectors. According to (1), the ensuing equation (15) existed. It is obvious that the minimum virtual gap $\Delta_{o}^{*}$ is equivalent to $\delta_{o}^{*}$. Referring to (16), this result ensures the nearest improvement target is found. The maximum total of improvement ratios in (16) ensures the improvement target is located on the envelopment.

$$
\begin{aligned}
1 & =\frac{\left(\sum_{r \in R} y_{r o} u_{r}^{*}+\Delta_{o}^{*}\right)}{\sum_{i \in I} x_{i o} v_{i}^{*}}, \\
\theta_{o}^{*} & =\frac{\sum_{r \in R} y_{r o} u_{r}^{*}}{\sum_{i \in I} x_{i o} v_{i}^{*}} .
\end{aligned}
$$

\section{Proposed Network Structure of VGM}

3.1. Network Structure. The network contains a set of subprocesses (nodes), $H$. The nodes are assigned ordinal numbers $1,2,3, \ldots,\|H\|$. Let $A$ denote the set of network links. There are $n$ homogeneous DMUs in set $J$, named $\mathrm{DMU}_{1}, \mathrm{DMU}_{2}, \ldots$, and $\mathrm{DMU}_{n}$, which are randomly processed by the subprocesses in set $H$.

3.1.1. Inputs and Outputs. At each subprocess $h$, there is a set of input measures $I^{h}$ that flow into the network and a set of output measures $R^{h}$ that flow out of the network. For $\mathrm{DMU}_{j}$ in set $J$, let $x_{i j}^{h} \in \mathfrak{R}_{+}^{I^{h}}$ and $y_{r j}^{h} \in \mathfrak{R}_{+}^{R^{h}}$ denote the volumes of the $i$ th input measure and the $r$ th output measure at subprocess $h$, respectively. Let $q_{i}^{h}$ and $p_{r}^{h}$ be the slack of the $i$ th input and the $r$ th output at subprocess $h$, respectively.

3.1.2. Links. Each subprocess may have links to other subprocesses. Let $(h, k)$ denote the link between subprocesses $h$ and $k, h>k$. Let $D^{(h, k)}$ denote the set of link measures on link $(h, k) \cdot z_{d j}^{(h, k)} \in \mathfrak{R}_{+}^{D^{(h, k)+}} \cup \mathfrak{R}_{+}^{D^{(h, k)-}}$ denotes the volume of the $d$ th link in set $D^{(h, k)}$. Each DMU alternatively acts as the DMU that is under evaluation. The volume of link $d$ on link $(h, k)$, $z_{d o}^{(h, k)}$, could be increased or decreased with a slack $f_{d}^{(h, k)}$ to improve the efficiency of $\mathrm{DMU}_{o}$ as well.

In Phase-I, we introduce the mixed binary integer virtual gap-based measurement model [M3] to partition the links into two subsets: as-input and as-output.

[M3] is as follows:

$$
\begin{aligned}
\delta_{o}^{(\mathrm{I}) *}=\max \quad & \tau^{\prime} \sum_{h \in H}\left(\sum_{i \in I^{h}} \frac{q_{i}^{h}}{x_{i o}^{h}}+\sum_{r \in R^{h}} \frac{p_{r}^{h}}{y_{r o}^{h}}+\sum_{(h, k) \in A} \sum_{d \in D^{(h, k)}} \frac{f_{d}^{(h, k)}}{z_{d o}^{(h, k)}}\right) ; \\
\text { s.t. } \quad & \sum_{j \in J} \pi_{j}^{h} x_{i j}^{h}=x_{i o}^{h}-q_{i}^{h}, \quad i \in I^{h} ; h \in H ; \\
& \sum_{j \in J} \pi_{j}^{h} y_{r j}^{h}=y_{r o}^{h}+p_{r}^{h}, \quad r \in R^{h}, h \in H ; \\
& \sum_{j \in J} \pi_{j}^{h} z_{d j}^{(h, k)}=z_{d o}^{(h, k)}-f_{d}^{(h, k)}+M t_{d}^{(h, k)}, \quad d \in D^{(h, k)},(h, k) \in A ; h \in H ; \\
& \sum_{j \in J} \pi_{j}^{h} z_{d j}^{(h, k)}=z_{d o}^{(h, k)}+f_{d}^{(h, k)}-M\left(1-t_{d}^{(h, k)}\right), \quad d \in D^{(h, k)},(h, k) \in A ; h \in H ; \\
& \sum_{j \in J}\left(\pi_{j}^{h}-\pi_{j}^{k}\right) z_{d j}^{(h, k)}=0, \quad d \in D^{(h, k)},(h, k) \in A ; h \in H ;
\end{aligned}
$$




$$
\begin{aligned}
& \pi_{j}^{h} \geq 0, \quad j \in J ; h \in H ; \\
& q_{j}^{h} \geq 0, \quad j \in J ; h \in H ; \\
& p_{j}^{h} \geq 0, \quad j \in J ; h \in H ; \\
& t_{d o}^{(h, k)} \in\{0,1\}, \quad d \in D^{(h, k)},(h, k) \in A, h \in H .
\end{aligned}
$$

In (17), the objective function maximizes the total improvement ratios of each input, output, and link of $\mathrm{DMU}_{o}$. The decision variable $\pi_{j}^{h}$ is the weight of $\mathrm{DMU}_{j}$ at subprocess $h$. The set of left-side of inequalities (18) (22) is the efficient frontier with respect to $\mathrm{DMU}_{o}$. The right-side of (18) is the improved $i$ th input at subprocess $h$ located on the frontier. The right-side of (19) is the improved $r$ th output at subprocess $h$ located on the frontier. $M$ denotes a considerably large constant. When the decision variable $t_{d o}^{(h, k)}=0,(20)$ becomes effective and (21) becomes ineffective and this constrain could be ignored, and the $d$ th measurement on link $D^{(h, k)}$ is treated as "as-input" with respect to the network. Conversely, when $t_{d o}^{(h, k)}=1,(20)$ becomes ineffective and (21) becomes effective, and the $d$ th measurement on link $D^{(h, k)}$ is treated as "asoutput" with respect to the network.

When $t_{d o}^{(h, k)}=0,(27)$ and (28) derived from (20) and (22) would ensure the improved $d$ th as-input at link $(h, k)$ projects on the frontier, respectively, to subprocesses $h$ and $k$ with the same value, $z_{d o}^{(h, k)}-f_{d}^{(h, k)}$.

$$
\begin{aligned}
& \sum_{j \in J} \pi_{j}^{h} z_{d j}^{(h, k)}=z_{d o}^{(h, k)}-f_{d}^{(h, k)}, \\
& d \in D^{(h, k)},(h, k) \in A ; h \in H ; \\
& \sum_{j \in J} \pi_{j}^{k} z_{d j}^{(h, k)}=z_{d o}^{(h, k)}-f_{d}^{(h, k)}, \\
& d \in D^{(h, k)},(h, k) \in A ; h \in H .
\end{aligned}
$$

When $t_{d o}^{(h, k)}=1$, the two equations (29) and (30) derived from (21) and (22) would ensure the deteriorated $d$ th asoutput at link $(h, k)$ projects on the frontier, respectively, to subprocesses $h$ and $k$ with the same value, $z_{d o}^{(h, k)}+f_{d}^{(h, k)}$.

$$
\sum_{j \in J} \pi_{j}^{h} z_{d j}^{(h, k)}=z_{d o}^{(h, k)}+f_{d}^{(h, k)}
$$

$$
\begin{aligned}
& \sum_{j \in J} \pi_{j}^{k} z_{d j}^{(h, k)}=z_{d o}^{(h, k)}+f_{d}^{(h, k)}, \\
& \quad d \in D^{(h, k)},(h, k) \in A ; h \in H .
\end{aligned}
$$

Therefore, a single constraint (22) would replace the two cases above (Chen et al. [20]).

Proposition 1. If it is an as-output direction for subprocess $h$, then it must be an as-input direction for subprocess $k$.

Proof. When $t_{d o}^{(h, k)}=0$, equations (20) and (22) are partitioned to (27) and (28) which is explained in prior section. If (28) is multiplied by -1 on each side of the equation, due to the fact that $z_{d j}^{(h, k)}=-z_{d j}^{(k, h)}$ and $f_{d}^{(h, k)}=f_{d}^{(k, h)}$, the modified (28) can be converted to (31):

$$
\begin{aligned}
& \sum_{j \in J} \pi_{j}^{k} z_{d j}^{(k, h)}=z_{d o}^{(k, h)}+f_{d}^{(k, h)}, \\
& \quad d \in D^{(k, h)},(k, h) \in A ; h \in H .
\end{aligned}
$$

For (27), from $\pi_{j}^{h}$ perspective, the links are as-input. For (31), from $\pi_{j}^{k}$ perspective, the links are as-output. With respect to (27) and (31), the direction is from subprocess $k$ to subprocess h.

Solve [M3] to obtain the optimal solutions of the binary integer variables. If $t_{d o}^{(h, k) *}=0$, then assign index $d$ to the set "as-input" that regards the entire system. Conversely, if $t_{d o}^{(h, k) *}=1$, then assign index $d$ to the set "as-output" that regards the entire system. On each link $(h, k)$, the set of links on $D^{(h, k)}$ is then partitioned into two subsets, $D_{o}^{(h, k)-}=\{d \mid$ $\left.t_{d o}^{(h, k) *}=0\right\}$ and $D_{o}^{(h, k)+}=\left\{d \mid t_{d o}^{(h, k) *}=1\right\}$. The notation $f_{d}^{(h, k)}$ in [M3] is replaced by $f_{d}^{(h, k)+}$ and $f_{d}^{(h, k)-}$ if $d \in D_{o}^{(h, k)+}$ and $d \in D_{o}^{(h, k)-}$, respectively. Then, the following linear program [M4] is constructed as Phase-II of the solving procedure:

$$
d \in D^{(h, k)},(h, k) \in A ; h \in H
$$

$$
\begin{gathered}
\delta_{o}^{(\mathrm{II}) *}=\max \tau^{\prime} \sum_{h \in H}\left(\sum_{i \in I^{h}} \frac{q_{i}^{h}}{x_{i o}^{h}}+\sum_{r \in R^{h}} \frac{p_{r}^{h}}{y_{r o}^{h}}+\sum_{(h, k) \in A}\left(\sum_{d \in D_{o}^{(h, k)-}} \frac{f_{d}^{(h, k)-}}{z_{d o}^{(h, k)}}+\sum_{d \in D_{o}^{(h, k)+}} \frac{f_{d}^{(h, k)+}}{z_{d o}^{(h, k)}}\right)\right) \\
\text { s.t. } \quad \sum_{j \in J} \pi_{j}^{h} x_{i j}^{h}=x_{i o}^{h}-q_{i}^{h}, \quad i \in I^{h}, h \in H ;
\end{gathered}
$$




$$
\begin{aligned}
& \sum_{j \in J} \pi_{j}^{h} y_{r j}^{h}=y_{r o}^{h}+p_{r}^{h}, \quad r \in R^{h}, h \in H ; \\
& \sum_{j \in J} \pi_{j}^{h} z_{d j}^{(h, k)}=z_{d o}^{(h, k)}-f_{d}^{(h, k)-}, \quad d \in D_{o}^{(h, k)-},(h, k) \in A ; h \in H ; \\
& \sum_{j \in J} \pi_{j}^{h} z_{d j}^{(h, k)}=z_{d o}^{(h, k)}+f_{d}^{(h, k)+}, \quad d \in D_{o}^{(h, k)+},(h, k) \in A ; h \in H ; \\
& \sum_{j \in J}\left(\pi_{j}^{h}-\pi_{j}^{k}\right) z_{d j}^{(h, k)}=0, \quad d \in D_{o}^{(h, k)-},(h, k) \in A ; h \in H ; \\
& \sum_{j \in J}\left(\pi_{j}^{h}-\pi_{j}^{k}\right) z_{d j}^{(h, k)}=0, \quad d \in D_{o}^{(h, k)+},(h, k) \in A ; h \in H ; \\
& \pi_{j}^{h} \geq 0, \quad j \in J ; h \in H ; \\
& q_{i}^{h} \geq 0, \quad i \in I ; h \in H ; \\
& p_{r}^{h} \geq 0, \quad r \in R ; h \in H ; \\
& f_{d}^{(h, k)+} \geq 0, \quad d \in D_{o}^{(h, k)+},(h, k) \in A ; h \in H ; \\
& f_{d}^{(h, k)-} \geq 0, \quad d \in D_{o}^{(h, k)-},(h, k) \in A ; h \in H .
\end{aligned}
$$

The objective function (32) expresses the maximum summation of improvement ratios of inputs, outputs, as-inputs, and as-outputs. Set $\tau^{\prime}=1$ for convenient computation and it would be adjusted according to a normalization process. Constraints (33), (34), (35), and (36) ensure the modified values of inputs, outputs, as-inputs, and as-outputs would project on the efficient frontier, respectively. Constraints (37) and (38) ensure the modified as-input and as-output links would project on the efficient frontier (Chen et al. [20]). Assign dual variables $v_{i}^{\prime h}, u_{r}^{\prime h}, w_{d}^{\prime(h, k)-}, w_{d}^{\prime(h, k)+}, \varsigma_{d}^{\prime(h, k)-}$, and $\varsigma_{d}^{\prime(h, k)+}$ to the functional constraints $(33) \sim(38)$, respectively. The transformed dual form [M5] is shown below.

$$
\begin{aligned}
& \text { [M5] } \Delta_{o}^{\prime *}=\min \sum_{h \in H}\left[\left(\sum_{i \in I^{h}} x_{i o}^{h} v_{i}^{\prime h}+\sum_{(h, k) \in A} \sum_{d \in D_{o}^{(h, k)-}} z_{d o}^{(h, k)} w_{d}^{\prime(h, k)-}\right)-\left(\sum_{r \in R^{h}} y_{r o}^{h} u_{r}^{\prime h}+\sum_{(h, k) \in A} \sum_{d \in D_{o}^{(h, k)+}} z_{d o}^{(h, k)} w_{d}^{\prime(h, k)+}\right)\right] \\
& \text { s.t. } \sum_{h \in H}\left[\left(\sum_{i \in I^{h}} x_{i j}^{h} v_{i}^{\prime h}+\sum_{(h, k) \in A} \sum_{d \in D_{o}^{(h, k)-}} z_{d j}^{(h, k)} w_{d}^{\prime(h, k)-}\right)-\left(\sum_{r \in R^{h}} y_{r j}^{h} u_{r}^{\prime h}+\sum_{(h, k) \in A} \sum_{d \in D_{o}^{(h, k)+}} z_{d j}^{(h, k)} w_{d}^{\prime(h, k)+}\right)\right. \\
&+\left(\sum_{(h, k) \in A} \sum_{d \in D_{o}^{(h, k)-}} z_{d j}^{(h, k)} \varsigma_{d}^{\prime(h, k)-}+\sum_{(h, k) \in A} \sum_{d \in D_{o}^{(h, k)+}} z_{d j}^{(h, k)} \varsigma_{d}^{\prime(h, k)+}\right) \\
&\left.-\left(\sum_{(k, h) \in A} \sum_{d \in D_{o}^{(k, h)-}} z_{d j}^{(k, h)} \varsigma_{d}^{\prime(k, h)-}+\sum_{(k, h) \in A} \sum_{d \in D_{o}^{(k, h)+}} z_{d j}^{(k, h)} \varsigma_{d}^{\prime(k, h)+}\right)\right] \geq 0 ; \\
& w_{d}^{\prime(h, k)+} z_{d o}^{(h, k)}=\tau^{\prime}, \quad d \in D_{o}^{(h, k)+},(h, k) \in A ; h \in H ; \\
& w_{d}^{\prime(h, k)-} z_{d o}^{(h, k)}=\tau^{\prime}, \quad d \in D_{o}^{(h, k)-},(h, k) \in A ; h \in H ; \\
& x_{i o}^{h} v_{i}^{\prime h} \geq \tau^{\prime}, \quad i \in I^{h}, h \in H ; \\
& y_{r o}^{h} u_{r}^{\prime h} \geq \tau^{\prime}, \quad r \in R^{h}, h \in H ; \\
& v_{i}^{\prime h} \geq 0, \quad i \in I^{h}, h \in H ;
\end{aligned}
$$




$$
\begin{aligned}
& u_{r}^{\prime h} \geq 0, \quad r \in R^{h}, h \in H ; \\
& w_{d}^{\prime(h, k)-} \geq 0, \quad d \in D_{o}^{(h, k)-},(h, k) \in A ; h \in H ; \\
& w_{d}^{\prime(h, k)+} \geq 0, \quad d \in D_{o}^{(h, k)+},(h, k) \in A ; h \in H ; \\
& \varsigma_{d}^{\prime(h, k)-} \text { free in sign, } d \in D_{o}^{(h, k)-},(h, k) \in A ; h \in H ; \\
& \varsigma_{d}^{\prime(h, k)+} \text { free in sign, } d \in D_{o}^{(h, k)+},(h, k) \in A ; h \in H ; \\
& \varsigma_{d}^{\prime(k, h)-} \text { free in sign, } d \in D_{o}^{(k, h)-},(k, h) \in A ; h \in H ; \\
& \varsigma_{d}^{\prime(k, h)+} \text { free in sign, } d \in D_{o}^{(k, h)+},(k, h) \in A ; h \in H .
\end{aligned}
$$

Replace the coefficient $\tau^{\prime}$ in VGM models by $\tau$, which is expressed as (57). Therefore, all the upper bound of normalized values of virtual-input plus virtual-as-input of DMUs is 1. Furthermore, all of the upper bound of normalized values of virtual-output plus virtual-as-output of DMUs is 1 , as well.

$$
\tau=\frac{1}{\max _{j \in J}\left[\sum_{h \in H}\left(\sum_{i \in I^{h}} x_{i j}^{h} v_{i}^{\prime h}+\sum_{(h, k) \in A} \sum_{d \in D_{o}^{(h, k)-}} z_{d j}^{(h, k)} w_{d}^{\prime(h, k)-}\right)\right]} .
$$

Then, the optimal solutions are normalized as

$$
\begin{aligned}
\Delta_{o}^{*} & =\Delta_{o}^{\prime *} \times \tau, \quad h \in H ; \\
v_{i}^{h *} & =v_{i}^{\prime h *} \times \tau, \quad i \in I^{h}, \quad h \in H ; \\
u_{r}^{h *} & =u_{r}^{\prime h *} \times \tau, \quad r \in R^{h}, h \in H ; \\
w_{d}^{(h, k)-*} & =w_{d}^{\prime(h, k)-*} \times \tau, \quad d \in D_{o}^{(h, k)-}, \quad(h, k) \in A ; \\
w_{d}^{(h, k)+*} & =w_{d}^{\prime(h, k)+*} \times \tau, \quad d \in D_{o}^{(h, k)+},(h, k) \in A ; \\
\varsigma_{d}^{(h, k)-*} & =\varsigma_{d}^{\prime(h, k)-*} \times \tau, \quad d \in D_{o}^{(h, k)-},(h, k) \in A ; \\
\varsigma_{d}^{(h, k)+*} & =\varsigma_{d}^{\prime(h, k)+*} \times \tau, \quad d \in D_{o}^{(h, k)+}, \quad(h, k) \in A .
\end{aligned}
$$

Evaluating different $\mathrm{DMU}_{o}$ 's, one may directly compare their weights, virtual gap, virtual-input, virtual-as-input, virtualas-output, and virtual-output vectors. According to (43), the ensuing equation (65) existed. It is obvious that the minimum virtual gap " $\Delta_{o}^{*}$ ” is equivalent to $\delta_{o}^{(\mathrm{II}) *}$. The maximum efficiency score of the entire network could be computed as (66).

1

$$
\begin{gathered}
=\frac{\sum_{h \in H}\left(\sum_{r \in R^{h}} y_{r o}^{h} u_{r}^{h *}+\sum_{(h, k) \in A} \sum_{d \in D_{o}^{(h, k)+}} z_{d o}^{(h, k)} w_{d}^{(h, k)+*}\right)+\Delta_{o}^{*}}{\sum_{h \in H}\left(\sum_{i \in I^{h}} x_{i o}^{h} v_{i}^{h *}+\sum_{(h, k) \in A} \sum_{d \in D_{o}^{(h, k)-}} z_{d o}^{(h, k)} w_{d}^{(h, k)-*}\right)} ; \\
E_{o}^{*}=\frac{\sum_{h \in H}\left(\sum_{r \in R^{h}} y_{r o}^{h} u_{r}^{h *}+\sum_{(h, k) \in A} \sum_{d \in D_{o}^{(h, k)+}} z_{d o}^{(h, k)} w_{d}^{(h, k)+*}\right)}{\sum_{h \in H}\left(\sum_{i \in I^{h}} x_{i o}^{h} v_{i}^{h *}+\sum_{(h, k) \in A} \sum_{d \in D_{o}^{(h, k)-}} z_{d o}^{(h, k)} w_{d}^{(h, k)-*}\right)} .
\end{gathered}
$$

3.2. Subprocess Efficiencies. (33) (36) depict the production technology of the network. The equations' right-side is the target on each index. At the improvement target, reducing the slacks $q_{i}^{h *}, i \in I^{h}, h \in H ; f_{d}^{(h, k)-*}, d \in D_{o}^{(h, k)-},(h, k) \in$ $A$ to the associated indices and adding the slacks $p_{r}^{h *}, r \in$ $R^{h}, h \in H ; f_{d}^{(h, k)+*}, d \in D_{o}^{(h, k)+},(h, k) \in A$ from the associated indices will improve the efficiency score to 1 . The efficient reference set of DMUs with respect to $\mathrm{DMU}_{o}$ is defined as $\mathrm{ER}_{o}=\left\{j \mid \pi_{j}^{h *}>0, h \in H, j \in J\right\}$. The efficiency score of subprocess $h$ is computed as follows:

$$
\begin{aligned}
& E_{o}^{h *} \\
& =\frac{\sum_{r \in R^{h}} y_{r o}^{h} u_{r}^{h *}+\sum_{(h, k) \in A} \sum_{d \in D_{o}^{(h, k)+}} z_{d o}^{(h, k)} w_{d}^{(h, k)+*}}{\sum_{i \in I^{h}} x_{i o}^{h} v_{i}^{h *}+\sum_{(h, k) \in A} \sum_{d \in D_{o}^{(h, k)-}} z_{d o}^{(h, k)} w_{d}^{(h, k)-*}} .
\end{aligned}
$$

3.3. Virtual Gap Diagram. For the virtual gap diagram, this paper defines the summation of input and as-input as $x$ axis $\left(\sum_{i \in I^{h}} x_{i o}^{h} v_{i}^{h *}+\sum_{(h, k) \in A} \sum_{d \in D_{o}^{(h, k)-}} z_{d o}^{(h, k)} w_{d}^{(h, k)-*}\right)$ and the summation of output and as-output as $y$-axis $\left(\sum_{r \in R^{h}} y_{r o}^{h} u_{r}^{h *}+\right.$ $\left.\sum_{(h, k) \in A} \sum_{d \in D_{o}^{(h, k)+}} z_{d o}^{(h, k)} w_{d}^{(h, k)+*}\right)$. For (66), it represents not only the maximum efficient score of the entire system but also the slope of the line from $\mathrm{DMU}_{o}$ to origin. From (65), if the virtual gap is zero, the optimal efficient score is 1 . We define the line with a slope equal to 1 to be the frontier. A larger virtual gap will entail a smaller slop and lower efficiency. Figure 1 depicts the $\mathrm{DMU}_{j}$ performance on the virtual gap diagram. The slope of $\mathrm{DMU}_{1}$ is 1 ; it is located on the efficiency frontier, indicating high efficiency. $\mathrm{DMU}_{2}$ slope is 0.6 , indicating lower efficiency; its location on the virtual 


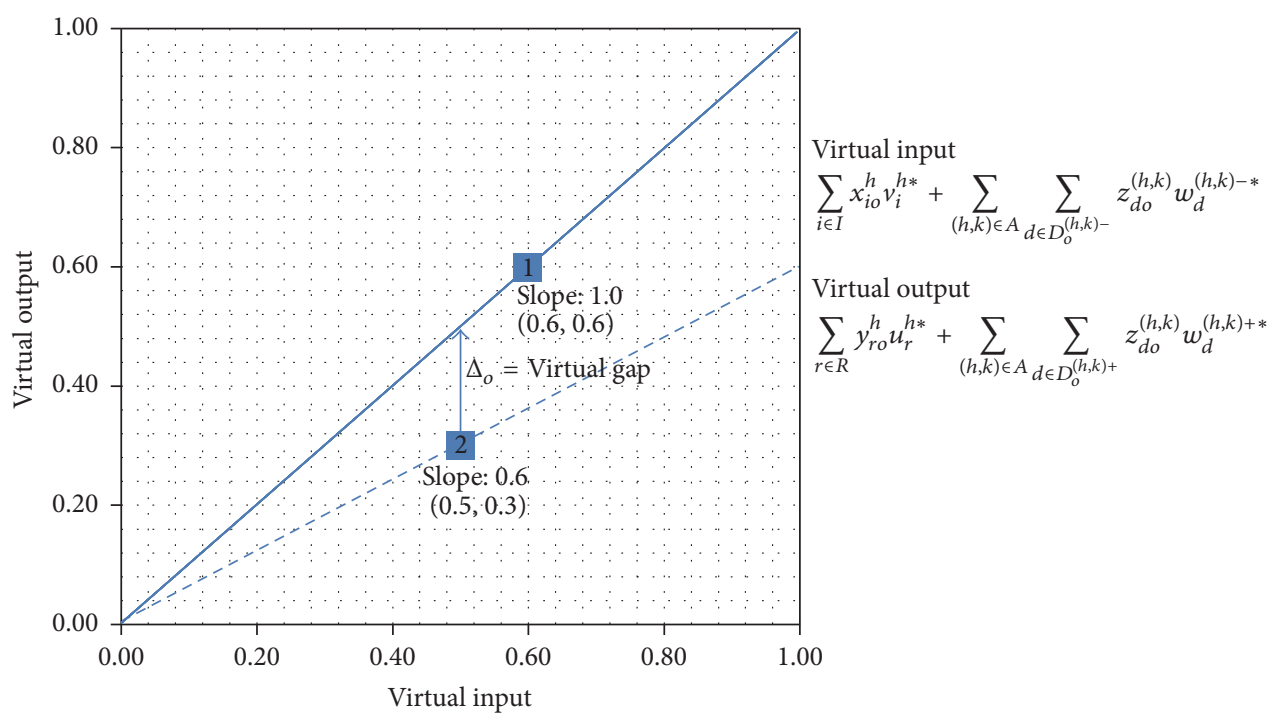

Figure 1: Virtual gap diagram.

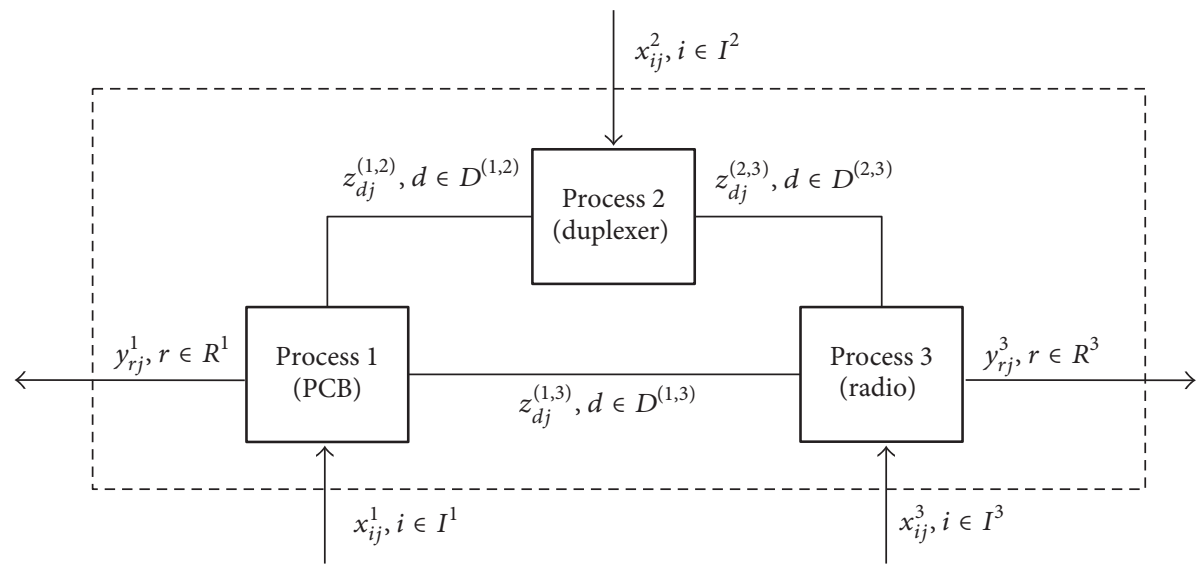

FIGURE 2: Example of network DEA.

gap diagram is $(0.5,0.3)$. For this $\mathrm{DMU}_{o}$, the virtual gap is 0.2 (0.5 minus 0.3 ). In order to improve the $\mathrm{DMU}_{o}$ efficiency, the virtual gap needs to be decreased.

\section{Illustrative Examples}

4.1. Data. This paper introduces a -realworld application of the network VGM model. One manufactory company produces microwave radio; this company owns three factories to produce printed circuit board (PCB), duplexer, and microwave radio. The relationship between the three factories is depicted in Figure 2; process 1 is PCB factory, process 2 is duplexer factory, and process 3 is microwave radio factory. Process 1 (PCB factory) purchases raw material from outside suppliers $\left(x_{i j}^{1}\right)$ and provides PCB to processes 2 and $3\left(z_{d j}^{(1,2)}\right.$ and $\left.z_{d j}^{(1,3)}\right)$. It also sells PCB to other customers $\left(y_{r j}^{1}\right)$. Process 2 (duplexer factory) purchases mechanical housing from other suppliers $\left(x_{i j}^{2}\right)$ and PCB from process $1\left(z_{d j}^{(1,2)}\right)$ to build duplexer and it sells duplexer to process $3\left(z_{d j}^{(2,3)}\right)$. Process
3 (microwave radio factory) purchases PCB from process 1 $\left(z_{d j}^{(1,3)}\right)$, duplexer from process $2\left(z_{d j}^{(2,3)}\right)$, and other materials $\left(x_{i j}^{3}\right)$ from other suppliers outside of this company to build microwave radio which it sells to end-users $\left(y_{r j}^{3}\right)$. Figure 2 depicts the entire network DEA system that is drawn as the rectangular dash box. The system contains a set of processes, $H=\{1,2,3\}$. The inputs, outputs, and links are shown in the figure. The set of links is $A=\{(1,2),(1,3),(2,3)\}$. For instance, the set of indices are $I^{1}=\{1\}, I^{2}=\{1\}$, $I^{3}=\{1\}, R^{1}=\{1\}, R^{2}=\{\}, R^{3}=\{1\}, D^{(1,2)}=\{1,2\}$, $D^{(1,3)}=\{1\}$, and $D^{(2,3)}=\{1,2\}$. The set of DMUs is $J=$ $\{1,2, \ldots, 11\}$. The arrows of inputs and outputs express the directions of their flows with respect to the system as well as to the processes. As regards the free link, the linking activities are freely determined while maintaining continuity between input and output. It demonstrates whether the current link flow is appropriate or needs to be increased or decreased.

Table 1 lists the hypothetical data of the indices of all DMUs. 
TABLE 1: The dataset of indices.

\begin{tabular}{lcccccccccc}
\hline $\mathrm{DMU}_{j}$ & $x_{1 j}^{1}$ & $x_{1 j}^{2}$ & $x_{1 j}^{3}$ & $z_{1 j}^{(1,2)}$ & $z_{2 j}^{(1,2)}$ & $z_{1 j}^{(1,3)}$ & $z_{1 j}^{(2,3)}$ & $z_{2 j}^{(2,3)}$ & $y_{1 j}^{1}$ & $y_{1 j}^{3}$ \\
\hline 1 & 5 & 10 & 714 & 20 & 43 & 538 & 74 & 6256 & 78 \\
2 & 6 & 32 & 786 & 20 & 65 & 773 & 84 & 6814 & 83 & 234 \\
3 & 5 & 25 & 851 & 25 & 60 & 612 & 70 & 9567 & 99 \\
4 & 5 & 30 & 544 & 24 & 60 & 591 & 64 & 7205 & 80 & 123 \\
5 & 9 & 13 & 700 & 30 & 70 & 547 & 98 & 4404 & 85 & 226 \\
6 & 9 & 41 & 678 & 27 & 58 & 592 & 72 & 6529 & 96 \\
7 & 5 & 33 & 948 & 12 & 59 & 587 & 85 & 6201 & 97 \\
8 & 6 & 10 & 658 & 13 & 42 & 540 & 70 & 8898 & 83 \\
9 & 5 & 41 & 641 & 10 & 46 & 676 & 73 & 4927 & 69 \\
10 & 9 & 20 & 885 & 28 & 60 & 659 & 79 & 6793 & 84 & 175 \\
11 & 6 & 25 & 727 & 22 & 41 & 655 & 84 & 4346 & 70 & 117 \\
\hline
\end{tabular}

TABLE 2: Partitions of links, as-input and as-output.

\begin{tabular}{|c|c|c|c|c|c|c|c|c|c|c|c|}
\hline \multirow{2}{*}{$\mathrm{DMU}_{o}$} & \multicolumn{5}{|c|}{$\begin{array}{c}\text { Binary decision variables for the five measures on } \\
\text { links }\end{array}$} & \multicolumn{6}{|c|}{ Sets of as-input and as-output on the three links } \\
\hline & $t_{10}^{(1,2) *}$ & $t_{20}^{(1,2) *}$ & $t_{10}^{(1,3) *}$ & $t_{10}^{(2,3) *}$ & $t_{20}^{(2,3) *}$ & $D_{o}^{(1,2)+}$ & $D_{o}^{(1,2)-}$ & $D_{o}^{(1,3)+}$ & $D_{o}^{(1,3)-}$ & $D_{o}^{(2,3)+}$ & $D_{o}^{(2,3)-}$ \\
\hline 1 & 0 & 1 & 0 & 1 & 1 & $\{2\}$ & $\{1\}$ & \{\} & $\{1\}$ & $\{1,2\}$ & \{\} \\
\hline 2 & 0 & 1 & 0 & 1 & 1 & $\{2\}$ & $\{1\}$ & \{\} & $\{1\}$ & $\{1,2\}$ & \{\} \\
\hline 3 & 1 & 0 & 0 & 1 & 0 & $\{1\}$ & $\{2\}$ & \{\} & $\{1\}$ & $\{1\}$ & $\{2\}$ \\
\hline 4 & 0 & 0 & 0 & 0 & 0 & \{\} & $\{1,2\}$ & \{\} & $\{1\}$ & \{\} & $\{1,2\}$ \\
\hline 5 & 0 & 0 & 1 & 0 & 1 & \{\} & $\{1,2\}$ & $\{1\}$ & \{\} & $\{2\}$ & $\{1\}$ \\
\hline 6 & 0 & 0 & 1 & 1 & 1 & \{\} & $\{1,2\}$ & $\{1\}$ & \{\} & $\{1,2\}$ & \{\} \\
\hline 7 & 1 & 0 & 1 & 0 & 0 & $\{1\}$ & $\{2\}$ & $\{1\}$ & \{\} & \{\} & $\{1,2\}$ \\
\hline 8 & 1 & 1 & 1 & 1 & 0 & $\{1,2\}$ & \{\} & $\{1\}$ & \{\} & $\{1\}$ & $\{2\}$ \\
\hline 9 & 1 & 1 & 0 & 1 & 1 & $\{1,2\}$ & \{\} & \{\} & $\{1\}$ & $\{1,2\}$ & \{\} \\
\hline 10 & 0 & 1 & 1 & 1 & 1 & $\{2\}$ & $\{1\}$ & $\{1\}$ & \{\} & $\{1,2\}$ & \{\} \\
\hline 11 & 0 & 1 & 0 & 1 & 1 & $\{2\}$ & $\{1\}$ & \{\} & $\{1\}$ & $\{1,2\}$ & \{\} \\
\hline
\end{tabular}

4.2. Results of VGM Case. Employ [M3] of this paper to solve the optimal solutions. Each row in Table 2 lists the optimal solutions of the binary variables. When $\mathrm{DMU}_{o}=$ $\mathrm{DMU}_{1}$, according to the first row, optimal values of five binary decision variables can be read as $0,1,0,1$, and 1 , and the six sets of as-input and as-output are $\{2\},\{1\},\{\},\{1\},\{1,2\}$, and \{\} . The decision variable $t_{1 o}^{(1,2) *}=0$ means that link 1 between processes 1 and 2 is treated as an as-input measure and $t_{1 o}^{(1,2) *}=1$ means that link 2 between processes 1 and 2 is treated as an as-output measure.

With the partitions of the links depicted in Table 2, one is ready to employ [M4]. The optimal values of the indices' slacks are listed in Table 3. When $\mathrm{DMU}_{1}$ is being evaluated, $\mathrm{DMU}_{o}=\mathrm{DMU}_{1}$ in the first column, all the slacks are zero. $\mathrm{DMU}_{1}$ is not dominated by the other DMUs, and it does not modify any values of indices. When $\mathrm{DMU}_{o}=\mathrm{DMU}_{3}$, in the third column, the optimal solution of [M4] decreases input values $x_{2}^{1}$ and $x_{2}^{2}$ by 3.286 and 25.411, respectively. The values of links $z_{1}^{(1,2)}, z_{2}^{(1,2)}, z_{1}^{(1,3)}, z_{1}^{(2,3)}$, and $z_{2}^{(2,3)}$ are modified by the values $-8.326,-2.078,-48.096,+14.863$, and -4651.133 , respectively. Increase output values $y_{1}^{3}$ by 3.169 .
Solving model [M4], one would also obtain the optimal values of dual variables as each DMU is played as $\mathrm{DMU}_{o}$. The optimal values of dual variables are listed and summarized in Table 4.

This paper adopts the coefficient $\tau$ (57) to limit the upper bound and normalize the optimal solution; $\tau$ is equal to the reciprocal of the maximum virtual-inputs of DMUs listed in Table 5.

According to (58) (64), the normalized weights of the indices are listed in Table 6.

The bottom rows of Tables 7 and 8 are the virtual-input and virtual-output of the DMUs, respectively. This paper defines a virtual gap diagram; the summation of input and as-input is the $x$-axis, and the summation of output and asoutput is the $y$-axis. Figure 3 depicts the locations of DMUs on this virtual gap diagram. $\mathrm{DMU}_{1}$ is located on the diagonal line because its efficiency score equals 1 . The other DMUs are located below the diagonal line because there is a virtual gap for each DMU.

Use (58) to calculate the solution of the virtual gap, $\Delta_{o}^{*}$. Use (66) to calculate the overall efficiency score of $\mathrm{DMU}_{o}, E_{o}^{*}$. Use (67) to calculate the subprocess efficiencies at process $h$, $E_{o}^{h *}, h=1,2,3$. Table 9 lists their values. 
TABLE 3: Slacks of the indices of every $\mathrm{DMU}_{o}$.

\begin{tabular}{|c|c|c|c|c|c|c|c|c|c|c|c|}
\hline \multirow{2}{*}{ Slacks } & \multicolumn{11}{|c|}{$\mathrm{DMU}_{o}$} \\
\hline & 1 & 2 & 3 & 4 & 5 & 6 & 7 & 8 & 9 & 10 & 11 \\
\hline$q_{1}^{1 *}$ & 0 & 0 & 3.286 & 0 & 0 & 3.644 & 0.822 & 0 & 0 & 0 & 2.618 \\
\hline$q_{1}^{2 *}$ & 0 & 11.289 & 25.411 & 21.713 & 18.914 & 0.822 & 19.009 & 0 & 21.267 & 11.035 & 3.242 \\
\hline$q_{1}^{3 *}$ & 0 & 101.510 & 0 & 0 & 0 & 0 & 0 & 0 & 197.015 & 0 & 0 \\
\hline$f_{1}^{(1,2)-*}$ & 0 & 0 & 8.326 & 4.877 & 13.241 & 14.168 & - & - & - & 3.846 & 6.215 \\
\hline$f_{1}^{(1,2)+*}$ & - & - & - & - & - & - & 3.111 & 10.077 & 12.672 & - & - \\
\hline$f_{2}^{(1,2)-*}$ & 0 & 0 & 2.078 & 15.380 & 13.937 & 18.851 & 10.436 & - & - & - & - \\
\hline$f_{2}^{(1,2)+*}$ & - & - & - & - & - & - & - & 11.846 & 0.270 & 17.651 & 10.383 \\
\hline$f_{1}^{(1,3)-*}$ & 0 & 0 & 48.096 & 50.473 & - & - & - & - & 21.746 & - & 122.544 \\
\hline$f_{1}^{(1,3)+*}$ & - & - & - & - & 85.253 & 63.823 & 171.004 & 143.581 & - & 31.949 & - \\
\hline$f_{1}^{(2,3)-*}$ & 0 & - & - & 1.532 & 5.011 & - & 6.939 & - & - & - & - \\
\hline$f_{1}^{(2,3)+*}$ & - & 12.374 & 14.836 & - & - & 12.752 & - & 5.385 & 2.689 & 13.752 & 38.305 \\
\hline$f_{2}^{(2,3)-*}$ & 0 & - & 4651.133 & 4397.778 & - & - & 4745.688 & 5510.308 & - & - & - \\
\hline$f_{2}^{(2,3)+*}$ & - & 5389.739 & - & - & 5477.336 & 6432.22 & - & - & 2412.840 & 8079.703 & 8118.121 \\
\hline$p_{1}^{1 *}$ & 0 & 0 & 0 & 0 & 12.075 & 0 & 7.369 & 9.794 & 0 & 21.783 & 15.000 \\
\hline$p_{1}^{3 *}$ & 0 & 80.803 & 3.169 & 4.781 & 9.085 & 0 & 0 & 26.768 & 64.298 & 112.870 & 80.000 \\
\hline
\end{tabular}

"_" means the decision variable is not in model [M4].

TABLE 4: Optimal weights of indices of model [M5].

\begin{tabular}{|c|c|c|c|c|c|c|c|c|c|c|c|}
\hline \multirow{2}{*}{ Weight } & \multicolumn{11}{|c|}{$\mathrm{DMU}_{o}$} \\
\hline & 1 & 2 & 3 & 4 & 5 & 6 & 7 & 8 & 9 & 10 & 11 \\
\hline$\overline{u_{1}^{\prime *}}$ & 0.053 & 0.012 & 0.271 & 0.075 & 0.025 & 0.043 & 0.053 & 0.012 & 0.014 & 0.004 & 0.014 \\
\hline$u_{3}^{\prime *}$ & 0.022 & 0.005 & 0.008 & 0.006 & 0.028 & 0.005 & 0.007 & 0.006 & 0.006 & 0.000 & 0.009 \\
\hline$v_{1}^{\prime *}$ & 0.200 & 0.167 & 4.953 & 0.288 & 0.111 & 0.111 & 1.190 & 0.199 & 0.789 & 0.111 & 0.236 \\
\hline$v_{2}^{\prime *}$ & 0.100 & 0.031 & 0.040 & 0.033 & 0.077 & 0.024 & 0.030 & 0.442 & 0.024 & 0.050 & 0.040 \\
\hline$v_{3}^{\prime *}$ & 0.001 & 0.002 & 0.001 & 0.003 & 0.012 & 0.009 & 0.001 & 0.003 & 0.006 & 0.005 & 0.007 \\
\hline$w_{1}^{\prime(1,2) *+}$ & - & - & - & - & - & - & 0.083 & 0.077 & 0.100 & - & - \\
\hline$w_{2}^{\prime(1,2) *+}$ & - & - & - & - & - & - & - & 0.024 & 0.022 & 0.017 & 0.024 \\
\hline$w_{1}^{\prime(1,3) *+}$ & - & - & - & - & 0.002 & 0.002 & 0.002 & 0.002 & - & 0.002 & - \\
\hline$w_{1}^{\prime(2,3) *+}$ & - & 0.012 & 0.014 & - & - & 0.014 & - & 0.014 & 0.014 & 0.013 & 0.012 \\
\hline$w_{2}^{\prime(2,3) *+}$ & - & 0.000 & - & - & 0.000 & 0.000 & - & - & 0.000 & 0.000 & 0.000 \\
\hline$w_{1}^{\prime(1,2) *-}$ & 0.050 & 0.050 & 0.040 & 0.042 & 0.033 & 0.037 & - & - & - & 0.036 & 0.045 \\
\hline$w_{2}^{\prime(1,2) *-}$ & 0.051 & 0.015 & 0.017 & 0.017 & 0.014 & 0.017 & 0.017 & - & - & - & - \\
\hline$w_{1}^{\prime(1,3) *-}$ & 0.002 & 0.001 & 0.002 & 0.002 & - & - & - & - & 0.001 & - & 0.002 \\
\hline$w_{1}^{\prime(2,3) *-}$ & 0.014 & - & - & 0.016 & 0.010 & - & 0.012 & - & - & - & - \\
\hline$w_{2}^{\prime(2,3) *-}$ & 0.000 & - & 0.000 & 0.000 & - & - & 0.000 & 0.000 & - & - & - \\
\hline$\varsigma_{1}^{\prime(1,2) *+}$ & 0.112 & 0.069 & 0.095 & 0.050 & 0.024 & 0.035 & 0.086 & - & - & 0.044 & 0.082 \\
\hline$\varsigma_{2}^{\prime(1,2) *+}$ & - & - & 0.121 & 0.063 & 0.015 & 0.038 & 0.092 & 0.044 & - & 0.016 & 0.016 \\
\hline$\varsigma_{1}^{\prime(1,3) *+}$ & - & 0.002 & 0.016 & 0.000 & - & - & 0.006 & - & 0.002 & - & 0.002 \\
\hline$\varsigma_{1}^{\prime(2,3) *+}$ & 0.090 & - & - & - & - & - & - & - & - & - & - \\
\hline$\varsigma_{2}^{\prime(2,3) *+}$ & - & - & - & - & - & - & - & - & - & - & - \\
\hline$\varsigma_{1}^{\prime(1,2) *-}$ & - & - & - & - & - & - & - & 0.068 & 0.068 & - & - \\
\hline$\varsigma_{2}^{\prime(1,2) *-}$ & 0.029 & 0.009 & - & - & - & - & - & - & 0.033 & - & - \\
\hline$\varsigma_{1}^{\prime(1,3) *-}$ & 0.002 & - & - & - & 0.000 & 0.001 & - & 0.000 & - & 0.001 & - \\
\hline$\varsigma_{1}^{\prime(2,3) *-}$ & - & 0.008 & 0.062 & 0.004 & 0.012 & 0.046 & 0.016 & 0.003 & 0.029 & 0.026 & 0.030 \\
\hline$\underline{\varsigma_{2}^{\prime(2,3) *-}}$ & 0.000 & 0.000 & 0.000 & 0.000 & 0.000 & 0.000 & 0.000 & 0.000 & 0.000 & 0.000 & 0.000 \\
\hline
\end{tabular}

TABLE 5: The coefficient $\tau$ for normalization.

$\mathrm{DMU}_{o}$

\begin{tabular}{|c|c|c|c|c|c|c|c|c|c|c|}
\hline 1 & 2 & 3 & 4 & 5 & 6 & 7 & 8 & 9 & 10 & 11 \\
\hline 0.069 & 0.144 & 0.020 & 0.090 & 0.060 & 0.084 & 0.064 & 0.045 & 0.073 & 0.140 & 0.089 \\
\hline
\end{tabular}


TABLE 6: Optimal weights of indices after normalization.

\begin{tabular}{|c|c|c|c|c|c|c|c|c|c|c|c|}
\hline \multirow{2}{*}{ Weight } & \multicolumn{11}{|c|}{$\mathrm{DMU}_{o}$} \\
\hline & 1 & 2 & 3 & 4 & 5 & 6 & 7 & 8 & 9 & 10 & 11 \\
\hline$u_{1}^{*}$ & 0.004 & 0.002 & 0.005 & 0.007 & 0.001 & 0.004 & 0.003 & 0.001 & 0.001 & 0.000 & 0.001 \\
\hline$u_{3}^{*}$ & 0.002 & 0.001 & 0.000 & 0.001 & 0.002 & 0.000 & 0.000 & 0.000 & 0.000 & 0.000 & 0.001 \\
\hline$v_{1}^{*}$ & 0.014 & 0.024 & 0.098 & 0.026 & 0.007 & 0.009 & 0.077 & 0.009 & 0.058 & 0.016 & 0.021 \\
\hline$v_{2}^{*}$ & 0.007 & 0.004 & 0.001 & 0.003 & 0.005 & 0.002 & 0.002 & 0.020 & 0.002 & 0.007 & 0.004 \\
\hline$v_{3}^{*}$ & 0.000 & 0.000 & 0.000 & 0.000 & 0.001 & 0.001 & 0.000 & 0.000 & 0.000 & 0.001 & 0.001 \\
\hline$w_{1}^{(1,2) *+}$ & - & - & - & - & - & - & 0.005 & 0.003 & 0.007 & - & - \\
\hline$w_{2}^{(1,2) *+}$ & - & - & - & - & - & - & - & 0.001 & 0.002 & 0.002 & 0.002 \\
\hline$w_{1}^{(1,3) *+}$ & - & - & - & - & 0.000 & 0.000 & 0.000 & 0.000 & - & 0.000 & - \\
\hline$w_{1}^{(2,3) *+}$ & - & 0.002 & 0.000 & - & - & 0.001 & - & 0.001 & 0.001 & 0.002 & 0.001 \\
\hline$w_{2}^{(2,3) *+}$ & - & 0.000 & - & - & 0.000 & 0.000 & - & - & 0.000 & 0.000 & 0.000 \\
\hline$w_{1}^{(1,2) *-}$ & 0.003 & 0.007 & 0.001 & 0.004 & 0.002 & 0.003 & - & - & - & 0.005 & 0.004 \\
\hline$w_{2}^{(1,2) *-}$ & 0.004 & 0.002 & 0.000 & 0.002 & 0.001 & 0.001 & 0.001 & - & - & - & - \\
\hline$w_{1}^{(1,3) *-}$ & 0.000 & 0.000 & 0.000 & 0.000 & - & - & - & - & 0.000 & - & 0.000 \\
\hline$w_{1}^{(2,3) *-}$ & 0.001 & - & - & 0.001 & 0.001 & - & 0.001 & - & - & - & - \\
\hline$w_{2}^{(2,3) *-}$ & 0.000 & - & 0.000 & 0.000 & - & - & 0.000 & 0.000 & - & - & - \\
\hline$\varsigma_{1}^{(1,2) *+}$ & 0.008 & 0.010 & 0.002 & 0.004 & 0.001 & 0.003 & 0.006 & - & - & 0.006 & 0.007 \\
\hline$\varsigma_{2}^{(1,2) *+}$ & - & - & 0.002 & 0.006 & 0.001 & 0.003 & 0.006 & 0.002 & - & 0.002 & 0.001 \\
\hline$\varsigma_{1}^{(1,3) *+}$ & - & 0.000 & 0.000 & 0.000 & - & - & 0.000 & - & 0.000 & - & 0.000 \\
\hline$\varsigma_{1}^{(2,3) *+}$ & 0.006 & - & - & - & - & - & - & - & - & - & - \\
\hline$\varsigma_{2}^{(2,3) *+}$ & - & - & - & - & - & - & - & - & - & - & - \\
\hline$\varsigma_{1}^{(1,2) *-}$ & - & - & - & - & - & - & - & 0.003 & 0.005 & - & - \\
\hline$\varsigma_{2}^{(1,2) *-}$ & 0.002 & 0.001 & - & - & - & - & - & - & 0.002 & - & - \\
\hline$\varsigma_{1}^{(1,3) *-}$ & 0.000 & - & - & - & 0.000 & 0.000 & - & 0.000 & - & 0.000 & - \\
\hline$\varsigma_{1}^{(2,3) *-}$ & - & 0.001 & 0.001 & 0.000 & 0.001 & 0.004 & 0.001 & 0.000 & 0.002 & 0.004 & 0.003 \\
\hline$\varsigma_{2}^{(2,3) *-}$ & 0.000 & 0.000 & 0.000 & 0.000 & 0.000 & 0.000 & 0.000 & 0.000 & 0.000 & 0.000 & 0.000 \\
\hline
\end{tabular}

TABLE 7: Input and as-input weighted data of the VGM model.

\begin{tabular}{|c|c|c|c|c|c|c|c|c|c|c|c|}
\hline \multirow{2}{*}{ Weight data } & \multicolumn{11}{|c|}{$\mathrm{DMU}_{o}$} \\
\hline & 1 & 2 & 3 & 4 & 5 & 6 & 7 & 8 & 9 & 10 & 11 \\
\hline$x_{1} v_{1}^{*}$ & 0.069 & 0.144 & 0.488 & 0.130 & 0.060 & 0.084 & 0.383 & 0.053 & 0.289 & 0.140 & 0.126 \\
\hline$x_{2} v_{2}^{*}$ & 0.069 & 0.144 & 0.020 & 0.090 & 0.060 & 0.084 & 0.064 & 0.198 & 0.073 & 0.140 & 0.089 \\
\hline$x_{3} v_{3}^{*}$ & 0.069 & 0.194 & 0.020 & 0.132 & 0.506 & 0.538 & 0.064 & 0.075 & 0.269 & 0.570 & 0.442 \\
\hline$z_{1}^{(1,2)} w_{1}^{(1,2)-*}$ & 0.069 & 0.144 & 0.020 & 0.090 & 0.060 & 0.084 & - & - & - & 0.140 & 0.089 \\
\hline$z_{2}^{(1,2)} w_{2}^{(1,2)-*}$ & 0.151 & 0.144 & 0.020 & 0.090 & 0.060 & 0.084 & 0.064 & - & - & - & - \\
\hline$z_{1}^{(1,3)} w_{1}^{(1,3)-*}$ & 0.069 & 0.144 & 0.020 & 0.090 & - & - & - & - & 0.073 & - & 0.089 \\
\hline$z_{1}^{(2,3)} w_{1}^{(2,3)-*}$ & 0.069 & - & - & 0.090 & 0.060 & - & 0.064 & - & - & - & - \\
\hline$z_{2}^{(2,3)} w_{2}^{(2,3)-*}$ & 0.083 & - & 0.020 & 0.090 & - & - & 0.064 & 0.045 & - & - & - \\
\hline $\begin{array}{l}\text { Summation }=\text { virtual } \\
\text { input }\end{array}$ & 0.648 & 0.913 & 0.607 & 0.802 & 0.808 & 0.873 & 0.704 & 0.370 & 0.705 & 0.991 & 0.834 \\
\hline
\end{tabular}

\section{Managerial Insights}

This paper adopts the VGM model to evaluate the indices of input items, output items, and links and identify the major efficiency improvement. As shown in Table 9, it indicates the efficiency scores of each subprocess. For $\mathrm{DMU}_{2}$, the actions needed to improve its low efficiency are subprocesses 1 and 2. The efficiency scores are 0.25 and 0.667 , respectively. For $\mathrm{DMU}_{11}$, the actions needed to improve the efficiency are to improve subprocesses 1 and 3 . The efficiency scores are 0.585 and 0.502 , respectively. If their efficiency scores are too low, even if subprocess 2 has higher performance, that will affect overall efficiency. The manager should review and consider the slacks of improvement, weight of each index, and virtual gap to define a plan and improve the efficiency of entire system.

The resulting VGM DEA scores provide complete information on how to improve the efficiency of DMUs for a 
TABLE 8: Output and as-output weighted data of the VGM model.

\begin{tabular}{|c|c|c|c|c|c|c|c|c|c|c|c|}
\hline \multirow{2}{*}{ Weight data } & \multicolumn{11}{|c|}{$\mathrm{DMU}_{o}$} \\
\hline & 1 & 2 & 3 & 4 & 5 & 6 & 7 & 8 & 9 & 10 & 11 \\
\hline$y_{1} u_{1}^{*}$ & 0.288 & 0.144 & 0.528 & 0.538 & 0.126 & 0.342 & 0.330 & 0.045 & 0.073 & 0.042 & 0.089 \\
\hline$y_{3} u_{3}^{*}$ & 0.360 & 0.174 & 0.020 & 0.090 & 0.385 & 0.084 & 0.064 & 0.045 & 0.073 & 0.000 & 0.089 \\
\hline$z_{1}^{(1,2)} w_{1}^{(1,2)+*}$ & - & - & - & - & - & - & 0.064 & 0.045 & 0.073 & - & - \\
\hline$z_{2}^{(1,2)} w_{2}^{(1,2)+*}$ & - & - & - & - & - & - & - & 0.045 & 0.073 & 0.140 & 0.089 \\
\hline$z_{1}^{(1,3)} w_{1}^{(1,3)+*}$ & - & - & - & - & 0.060 & 0.084 & 0.064 & 0.045 & - & 0.140 & - \\
\hline$z_{1}^{(2,3)} w_{1}^{(2,3)+*}$ & - & 0.144 & 0.020 & - & - & 0.084 & - & 0.045 & 0.073 & 0.140 & 0.089 \\
\hline$z_{2}^{(2,3)} w_{2}^{(2,3)+*}$ & - & 0.144 & - & - & 0.060 & 0.084 & - & - & 0.073 & 0.140 & 0.089 \\
\hline $\begin{array}{l}\text { Summation = virtual } \\
\text { output }\end{array}$ & 0.648 & 0.606 & 0.568 & 0.628 & 0.632 & 0.677 & 0.523 & 0.268 & 0.440 & 0.603 & 0.444 \\
\hline
\end{tabular}

TABLE 9: Efficiency and virtual gaps solved by VGM model.

\begin{tabular}{lccccccccccc}
\hline $\mathrm{DMU}_{j}$ & 1 & 2 & 3 & 4 & 5 & 6 & 7 & 8 & 9 & 10 \\
\hline Virtual input & 0.648 & 0.913 & 0.607 & 0.802 & 0.808 & 0.873 & 0.704 & 0.370 & 0.705 & 0.991 & 0.834 \\
Virtual output & 0.648 & 0.606 & 0.568 & 0.628 & 0.632 & 0.677 & 0.523 & 0.268 & 0.440 & 0.603 & 0.444 \\
Virtual gap & 0 & 0.307 & 0.039 & 0.174 & 0.176 & 0.196 & 0.181 & 0.102 & 0.265 & 0.470 & 0.390 \\
$E_{o}$ & 1.000 & 0.663 & 0.936 & 0.783 & 0.782 & 0.775 & 0.743 & 0.724 & 0.624 & 0.608 & 0.532 \\
$E_{o}^{1}$ & 0.804 & 0.250 & 0.965 & 1.344 & 1.029 & 1.697 & 1.026 & 3.357 & 0.606 & 1.148 & 0.585 \\
$E_{o}^{2}$ & 0 & 0.667 & 0.250 & 0 & 0.250 & 0.667 & 0.250 & 0.553 & 4.000 & 1.500 & 1.500 \\
$E_{o}^{3}$ & 1.242 & 1.368 & 0.667 & 0.224 & 0.893 & 0.622 & 0.667 & 1.121 & 0.642 & 0.738 & 0.502 \\
\hline
\end{tabular}

specific network process. The VGM impartially measures items with considerable measuring unit difference and is unit-invariant. The VGM can be applied in supply chain management which takes the perspective of organization mechanism to deal with the complex interactions in supply chain. The broadcasting company in Tone and Tsutsui [9] includes two departments: one is program department which produces programs and the other is transmission department which broadcasts programs. The links between the two departments are the program broadcasting plan which is generated from program producer. The producer would apply VGM and consider time, advertisement revenue, and customer preference to increase or decrease the program transmission.

\section{Discussion and Conclusions}

The contributions and innovative progress of this paper are that (1) it solves the MIP unsolvable nonlinear program model through a two-phase procedure by using a mixed integer program and (2) it creates a mathematical model and converts multiefficiency frontiers for the separation processes to an aggregation efficiency frontier for the entire production system, eventually obtaining the best-practice performance. The objective of efficiency assessment is to identify weaknesses such that the appropriate steps to improve the entire system performance can be taken. This paper introduces a two-phase procedure to evaluate the network DEA with "free" links. This new procedure employs VGM and considers not only the input and output slacks in the objective function but also the slacks of intermediate measures. The adjustment in the slacks of input, links, and output items defines the best-practice performance. The resulting DEA scores provide complete information on how to project inefficient DMUs onto the DEA frontier for a specific network DEA. The VGM impartially measures items with considerable measuring unit differences and is unit-invariant. Instead of the two conflicting roles that each link plays in existing models, each link plays a single role in the proposed network system in that it is either desirable or undesirable. We derived the dual method of the envelopment form, the multiplier form, to express how to obtain the weights of the as-input and as-output items. Each link is assigned a single weight. This procedure is similar to the legacy radial DEA models that determine the virtual weights of the inputs (as-input) and outputs (asoutput) of each $\mathrm{DMU}_{o}$. In computing the performance score, the signs of the as-input and as-output items are always opposite. The single assignment of weights for all of the performance indices, inputs, outputs, and links is crucial for performance analysis. The quantity of all process links may be considerably large. The current two-phase procedure is capable of solving the problem in nonpolynomial complexity. The new procedure will also be applied in series multistage, shared resource (Chen et al. [21] and Liang et al. [22]), dynamic network DEA (Tone and Tsutsui [10] and Kao [11]), assurance region (Thompson Jr. et al. [23]), cone ratio model (Charnes et al. [24]), and virtual weight analysis models (Sarrico and Dyson [25]) in future research.

\section{Competing Interests}

The authors declare that there is no conflict of interests regarding the publication of this paper. 


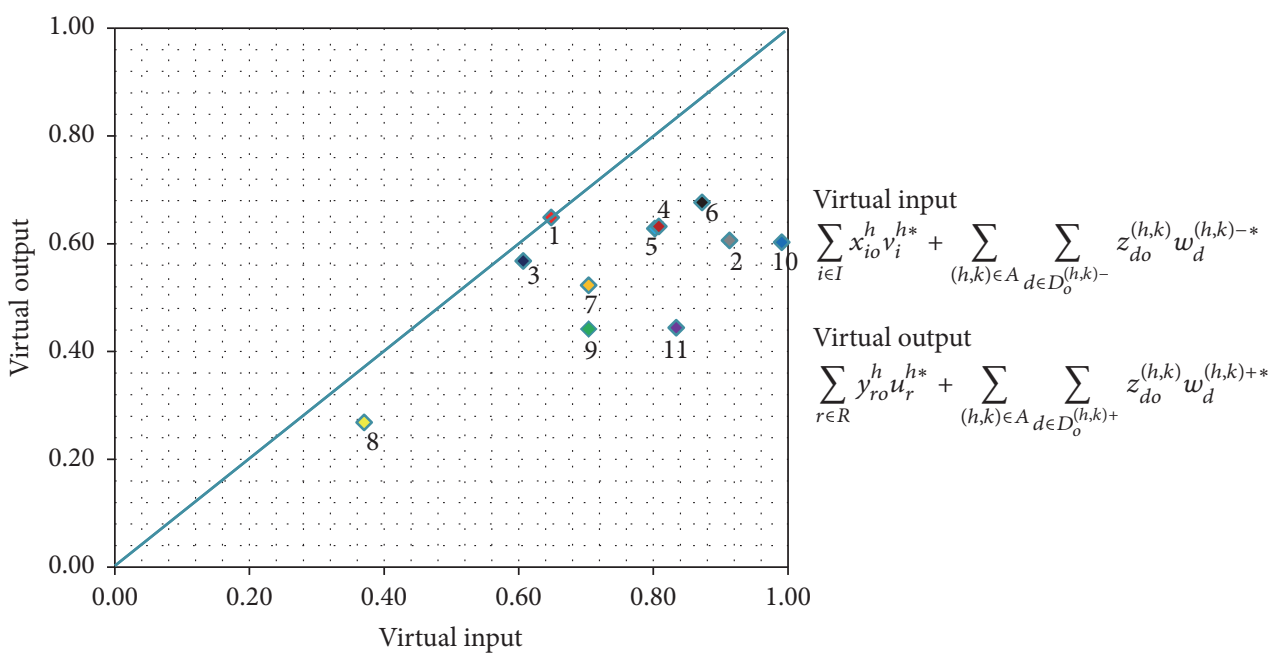

FIgURE 3: Locations of the 11 DMUs on the virtual gap diagram.

\section{Acknowledgments}

This research is supported by the Ministry of Science and Technology of Taiwan, Republic of China, under Project no. 100-2221-E-009-065-MY3.

\section{References}

[1] L. M. Seiford and J. Zhu, "Profitability and marketability of the top 55 U.S. commercial banks," Management Science, vol. 45, no. 9, pp. 1270-1288, 1999.

[2] J. Zhu, "Multi-factor performance measure model with an application to Fortune 500 companies," European Journal of Operational Research, vol. 123, no. 1, pp. 105-124, 2000.

[3] T. R. Sexton and H. F. Lewis, "Two-stage DEA: an application to major league baseball," Journal of Productivity Analysis, vol. 19, no. 2-3, pp. 227-249, 2003.

[4] H. F. Lewis and T. R. Sexton, "Network DEA: efficiency analysis of organizations with complex internal structure," Computers and Operations Research, vol. 31, no. 9, pp. 1365-1410, 2004.

[5] R. Färe and G. Whittaker, "An intermediate input model of dairy production using complex survey data," Journal of Agricultural Economics, vol. 46, no. 2, pp. 201-213, 1995.

[6] R. Färe and S. Grosskopf, "Productivity and intermediate products: a frontier approach," Economics Letters, vol. 50, no. 1, pp. 65-70, 1996.

[7] R. Färe and S. Grosskopf, "Intertemporal production frontiers: with dynamic DEA," Journal of the Operational Research Society, vol. 48 , no. 6, p. 656, 1997.

[8] C. Kao and S.-N. Hwang, "Efficiency decomposition in twostage data envelopment analysis: an application to non-life insurance companies in Taiwan," European Journal of Operational Research, vol. 185, no. 1, pp. 418-429, 2008.

[9] K. Tone and M. Tsutsui, "Network DEA: a slacks-based measure approach," European Journal of Operational Research, vol. 197, no. 1, pp. 243-252, 2009.

[10] K. Tone and M. Tsutsui, "Dynamic DEA with network structure: a slacks-based measure approach," Omega, vol. 42, no. 1, pp. 124131, 2014.
[11] C. Kao, "Dynamic data envelopment analysis: a relational analysis," European Journal of Operational Research, vol. 227, no. 2, pp. 325-330, 2013.

[12] K. Tone and M. Tsutsui, "Dynamic DEA: a slacks-based measure approach," Omega, vol. 38, no. 3-4, pp. 145-156, 2010.

[13] C. Kao, "Efficiency decomposition in network data envelopment analysis with slacks-based measures," Omega, vol. 45, pp. $1-6,2014$.

[14] Y. Cheng and H.-L. Gao, "Matrix-type network DEA model with its application based on input-output tables," Mathematical Problems in Engineering, vol. 2015, Article ID 505941, 8 pages, 2015.

[15] S. Lozano, "Alternative SBM model for network DEA," Computers \& Industrial Engineering, vol. 82, pp. 33-40, 2015.

[16] N. K. Avkiran, "An illustration of dynamic network DEA in commercial banking including robustness tests," Omega, vol. 55, pp. 141-150, 2015.

[17] J. Huang, J. Chen, and Z. Yin, "A network DEA model with super efficiency and undesirable outputs: an application to bank efficiency in China," Mathematical Problems in Engineering, vol. 2014, Article ID 793192, 14 pages, 2014.

[18] F. H. Liu and Y. C. Huang, "Virtual-gap measurement (VGM) for assessing a set of units," in Proceedings of the International Conference on Science, Technology, Engineering and Management (ICSTEM '15), Master thesis, 2015, http://www.ircsme .com/Programme\%20Schedule\%20-\%20IRCSME\%202015.pdf.

[19] W. D. Cook and J. Zhu, "Classifying inputs and outputs in data envelopment analysis," European Journal of Operational Research, vol. 180, no. 2, pp. 692-699, 2007.

[20] Y. Chen, W. D. Cook, and J. Zhu, "Deriving the DEA frontier for two-stage processes," European Journal of Operational Research, vol. 202, no. 1, pp. 138-142, 2010.

[21] Y. Chen, J. Du, H. D. Sherman, and J. Zhu, "DEA model with shared resources and efficiency decomposition," European Journal of Operational Research, vol. 207, no. 1, pp. 339-349, 2010.

[22] L. Liang, F. Yang, W. D. Cook, and J. Zhu, "DEA models for supply chain efficiency evaluation," Annals of Operations Research, vol. 145, no. 1, pp. 35-49, 2006. 
[23] R. G. Thompson Jr., F. D. Singleton, R. M. Thrall, and B. A. Smith, "Comparative site evaluations for locating a high-energy physics lab in Texas," Interfaces, vol. 16, no. 6, pp. 35-49, 1986.

[24] A. Charnes, W. W. Cooper, Z. M. Huang, and D. B. Sun, "Polyhedral Cone-Ratio DEA Models with an illustrative application to large commercial banks," Journal of Econometrics, vol. 46, no. 1-2, pp. 73-91, 1990.

[25] C. S. Sarrico and R. G. Dyson, "Restricting virtual weights in data envelopment analysis," European Journal of Operational Research, vol. 159, no. 1, pp. 17-34, 2004. 


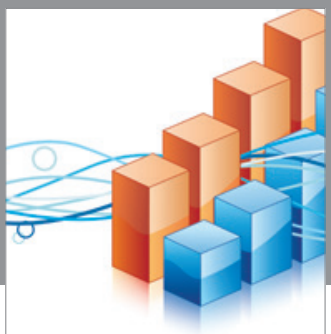

Advances in

Operations Research

vatem alat4

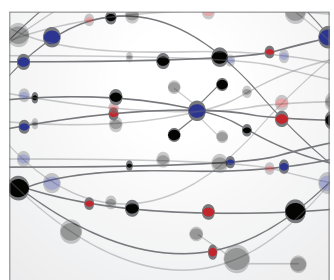

\section{The Scientific} World Journal
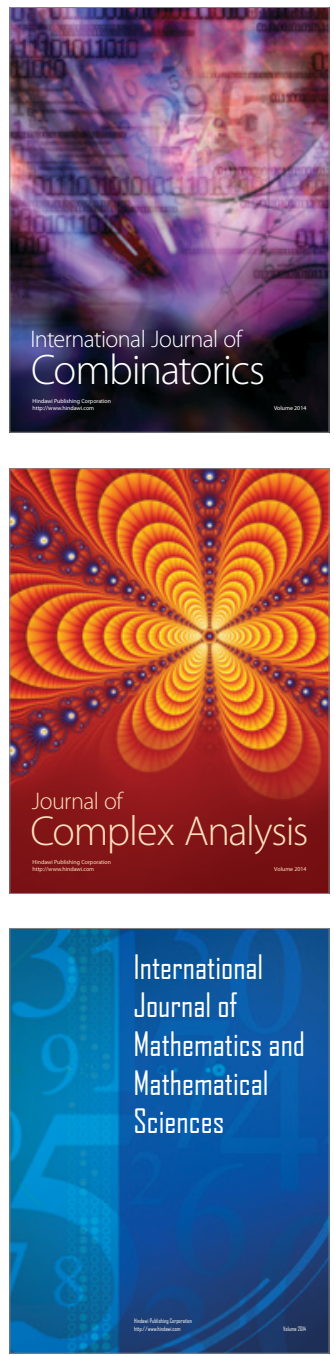
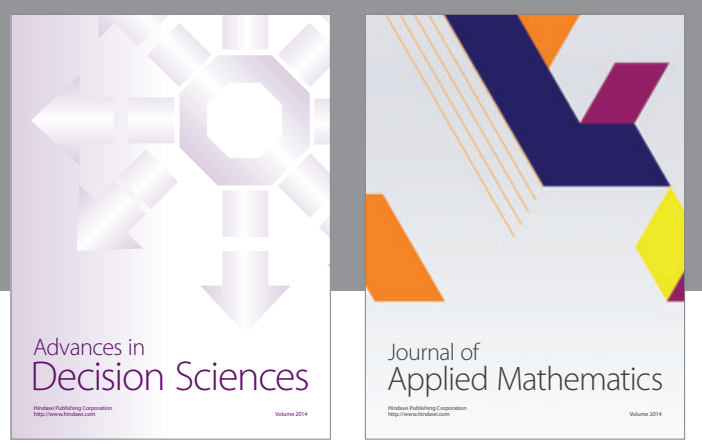

Algebra

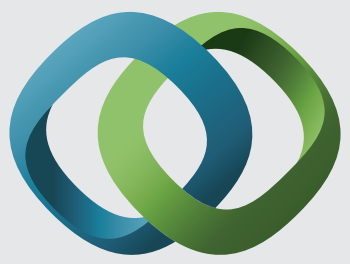

\section{Hindawi}

Submit your manuscripts at

https://www.hindawi.com
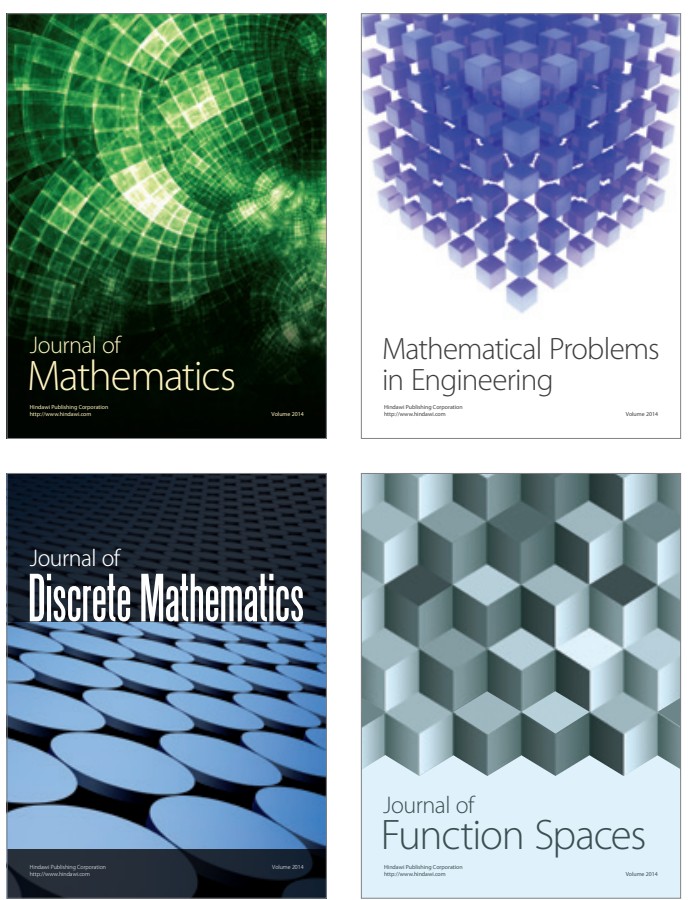

Mathematical Problems in Engineering
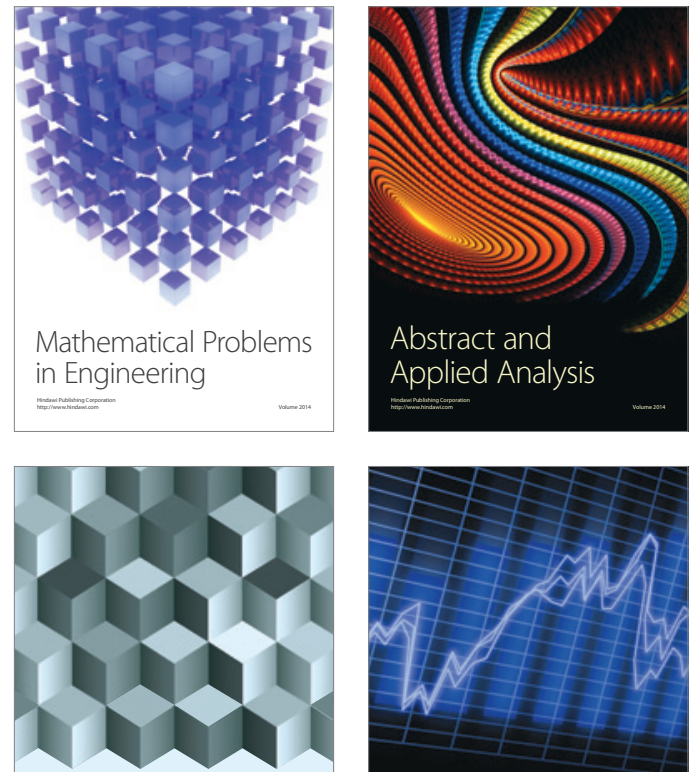

Journal of

Function Spaces

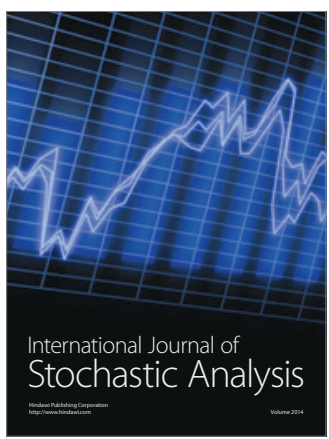

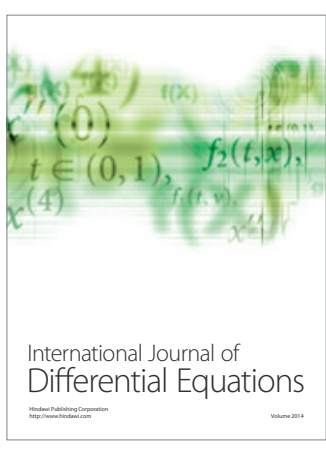
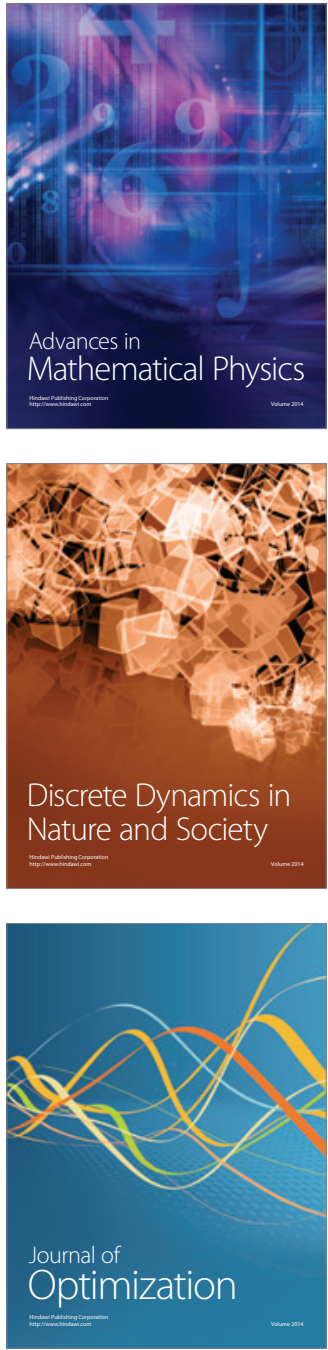